\title{
Step-induced magnetic-hysteresis anisotropy in ferromagnetic thin films
}

\author{
D. Zhao, Feng Liu, ${ }^{\text {a) }}$ D. L. Huber, and M. G. Lagallyb) \\ University of Wisconsin, Madison, Wisconsin 53706
}

(Received 5 September 2001; accepted for publication 15 November 2001)

\begin{abstract}
We investigate the quasistatic magnetic hysteresis of ferromagnetic thin films grown on a vicinal substrate, using Monte Carlo simulations within a two-dimensional $X Y$ model. Intrinsic in-plane anisotropy is assigned to surface sites according to their local symmetry. The simulated hysteresis loops show a strong anisotropy: the coercive field is the largest when the external field is along the step direction and vanishes when the external field is perpendicular to the step direction. In general, the coercivity increases with increasing step density, but displays a more complex dependence on film thickness. The simulations also suggest that the mechanism for the magnetization reversal is coherent rotation. These results are in good agreement with experiments. (C) 2002 American Institute of Physics. [DOI: 10.1063/1.1433179]
\end{abstract}

The magnetic properties of ferromagnetic thin films and multilayers have been extensively studied because of their potential impact on magnetic recording devices. As the thickness of a film is reduced, its properties are expected to be strongly influenced by surfaces and interfaces, which are inevitably rough at atomic scales. The ultimate goal of studies of the influences of surface/interface roughness on magnetic properties of thin films ${ }^{1-17}$ is to engineer desirable magnetic properties by artificially creating and controlling the surface/ interface structure and morphology.

Surface/interface roughness strongly influences both the static (e.g., magnetization) ${ }^{17}$ and dynamic (e.g., magnetic hysteresis ${ }^{16}$ magnetic properties of thin films. Experiments have demonstrated that surface steps induce an in-plane uniaxial magnetic anisotropy, with the easy axis parallel to the step direction, in a variety of magnetic thin films ${ }^{1-11}$ grown on metal as well as on semiconductor stepped surfaces. The measured hysteresis loops show that the coercive field decreases while the saturation field increases when the external field is turned away from the direction parallel to the steps to the direction perpendicular to the steps, and such uniaxial anisotropy increases with increasing step density. ${ }^{10}$ Theoretical modeling and simulations have been carried out to investigate the effect of steps on magnetization reversal. ${ }^{14-16}$ In particular, Hyman et al. ${ }^{16}$ have recently derived, within a two-dimensional $X Y$ model, a phase diagram of hysteresis loops in the parameter space of anisotropy strength and step density, for magnetic reversal on vicinal surfaces.

In this article, we carry out Monte Carlo simulations of hysteresis loops of ferromagnetic thin films to extend these earlier theoretical studies. ${ }^{14-16}$ Our focus is to investigate systematically the effect of step density and film thickness on magnetic hysteresis anisotropy in ultrathin films grown on vicinal substrates. Our simulations show that the coercive (saturation) field decreases (increases) monotonically as the

\footnotetext{
a) Present address:University of Utah, Salt Lake City, UT 84112; electronic mail:fliu@eng.utah.edu ${ }^{b}$ lagally@engr.wisc.edu
}

angle between the external field and the step direction is increases from $0^{\circ}$ to $90^{\circ}$, exhibiting a strong hysteresis anisotropy. The coercivity, for all the directions of the external field, increases with increasing step density for a given film thickness but shows a more complex dependence on film thickness for a given step density. Detailed inspection of the evolution of the spin configuration along the hysteresis loops reveals that the mechanism for the magnetization reversal in all the simulated systems is dominated by coherent rotation.

We consider magnetic thin films a few atomic layers thick grown on a vicinal substrate with a simple-cubiccrystal structure, as shown in Fig. 1. The broken symmetry at surfaces and steps introduces a fourfold anisotropy at a surface terrace site and a uniaxial anisotropy at a step-edge site $;^{18}$ the effect is enhanced in ultrathin films, in which the surface (interface)-to-volume ratio is high. Different degrees of surface roughness are constructed by changing the surface step density and the film thickness. In order to reveal the dependence of hysteresis anisotropy on surface step density and on film thickness, we simulate, for each given sample configuration, hysteresis loops as a function of the angle between the external field and the step direction. In the present work, we limit our study to low temperature and small frequency (i.e., slowly varying external field), which corresponds to the "static scalar hysteresis" limit. ${ }^{19}$

We adopt a simple two-dimensional (2D) $X Y$ model $^{14}$ in describing the system. The Hamiltonian is given by

$$
\begin{aligned}
H= & -J \sum_{\langle i, j\rangle}\left(S_{i}^{x} S_{j}^{x}+S_{i}^{y} S_{j}^{y}\right)-\sum_{i} K_{2}^{i} S_{i} \cos ^{2}\left(\theta_{i}\right) \\
& -\sum_{i} K_{4}^{i} S_{i} \cos ^{2}\left(2 \theta_{i}\right)-h \sum_{i} S_{i} \cos \left(\theta_{i}-\phi\right),
\end{aligned}
$$

where $\theta_{i}$ is the angle between the direction of the vector spin $S_{i}$ and the [100] lattice direction, and $\left|S_{i}\right|=1 ; J$ is the exchange coupling between nearest-neighbor spins, $S_{i}$ and $S_{j}$. $K_{2}^{i}$ and $K_{4}^{i}$ represent the strength of uniaxial and fourfold anisotropy for a step and a terrace spin, respectively. The uniaxial anisotropy is along the [100] direction (parallel to the steps), and the fourfold anisotropy is along [100] and 


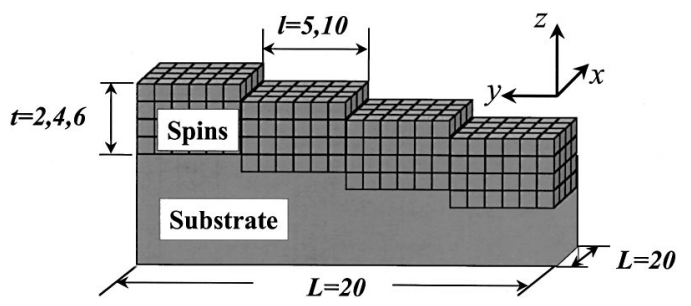

FIG. 1. Schematic diagram of simulation samples. The sample size is chosen to be $20 \times 20 \times t$, where $t$ is the thickness of the film chosen to be two, four, and six atomic layers $(t=2,4$, and 6 , each with two step densities $s=1 / 5$ and $1 / 10$ ). Periodic boundary conditions are used in the plane of the film surface and free boundary conditions are used in the direction normal to surface. The interaction between the substrate and the magnetic film is ignored.

[010] directions. The external magnetic field with strength $h$ lies in the $(\mathrm{x}, \mathrm{y})$ surface plane with an angle $\phi$ from [100]. For simplicity, all the terms in Eq. (1) are renormalized to be dimensionless by expressing energy in reduced units of $J(J$ $=1$ ) and setting the lattice spacing to unity. In the simulation, we choose parameters $K_{2}^{i}=0.1$ and $K_{4}^{i}=10^{-3}$, as used by Moschel et al., ${ }^{14}$ and consistent with experimental values. ${ }^{18}$ Periodic boundary conditions are used in the $x$ and $y$ directions and free boundary conditions in the $z$ direction (normal to the surface).

In order to simulate the equilibrium magnetization under a given field, we first determine the relaxation time for each system. To do so, a system is first relaxed under an external field of the maximum strength $(h=2)$ until equilibrium is reached. The direction of the field is then reversed: the time required for the system to reach equilibrium again defines the relaxation time. Next, we calculate the hysteresis loop by a series of Monte Carlo simulations. Starting with a spin lattice with a ferromagnetic structure, we first relax the system under a uniform external magnetic field until equilibrium is reached [typically about 5000 Monte Carlo steps (MCS)]. We then decrease the external field linearly from a given value in one direction to the same value in the opposite direction and calculate the magnetization along the path of changing external field. We change the external field in small steps of 0.01 and use a time duration between steps of $2 \tau$ MCS, where $\tau$ is the relaxation time of the film. This extremely slowly varying rate corresponds to a very small frequency of a sinusoidally varying external field. This choice should guarantee a proper quasistatic study, ${ }^{19}$ as an almost constant hysteresis loop shape is experimentally observed at very low frequencies. ${ }^{6}$ The Curie temperature for a 3D cubic $X Y$ spin lattice is $\sim 2.2 .{ }^{20}$ Our simulations are carried out at a system temperature of 0.5 , which lies in a wellordered ferromagnetic phase and is thus comparable to the experimental conditions.

All the results presented here are obtained from a lattice size of $20 \times 20 \times t$, where $t$ is the thickness of the film. (A few larger sizes up to $100 \times 100 \times t$ are also tested to ensure convergence with respect to the finite-size effect.) We consider three different thicknesses $(t=2,4$, and 6$)$, each with two step densities $(s=1 / 5$ and $1 / 10)$. For each system size, the hysteresis is calculated with six different angles between the direction of the external field and the direction of the
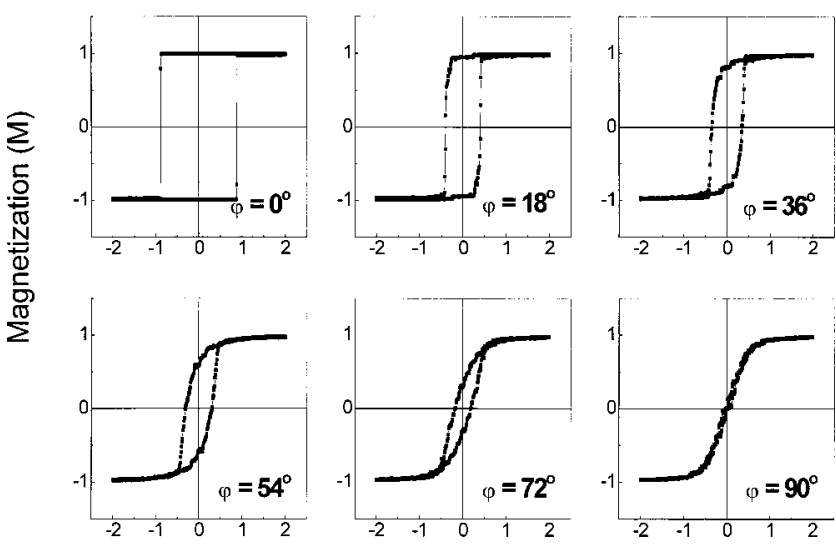

External Field $(\mathrm{H})$

FIG. 2. Simulated hysteresis loops for $t=4$ and $s=1 / 5$, at different angles, $\phi$, between the direction of the external field and the step direction: $\phi=0^{\circ}$, $18^{\circ}, 36^{\circ}, 54^{\circ}, 72^{\circ}$, and $90^{\circ}$.

steps $\left(\phi=0^{\circ}, 18^{\circ}, 36^{\circ}, 54^{\circ}, 72^{\circ}\right.$, and $\left.90^{\circ}\right)$. Below, we discuss the calculated hysteresis in terms of all three variables, $t, s$, and $\phi$.

Figure 2 shows typical hysteresis loops at different angles of $\phi$, for a film thickness $t=4$ and a step density of $s=1 / 5$, demonstrating the strong hysteresis anisotropy. As the angle between the external field and the step direction is increased from $\phi=0^{\circ}$ to $\phi=90^{\circ}$, the coercive field $H_{c}$ decreases from the largest value of 0.89 , when the field is along the step direction (easy axis), to almost 0 , when the field is perpendicular to the step direction. Concurrently, the saturation field increases from $H_{s}=0.89$ to $H_{s} \sim 2.0$; the remanence magnetization decreases from 1 to 0 as the squareness of the shape of hysteresis loops decreases. All the changes are monotonic as a function of $\phi$ (see Figs. 3 and 4) and the same behavior is observed in all six systems studied.

Figure 3 shows the dependence of coercivity (as a function of $\phi$ ) on step density for three different film thicknesses.

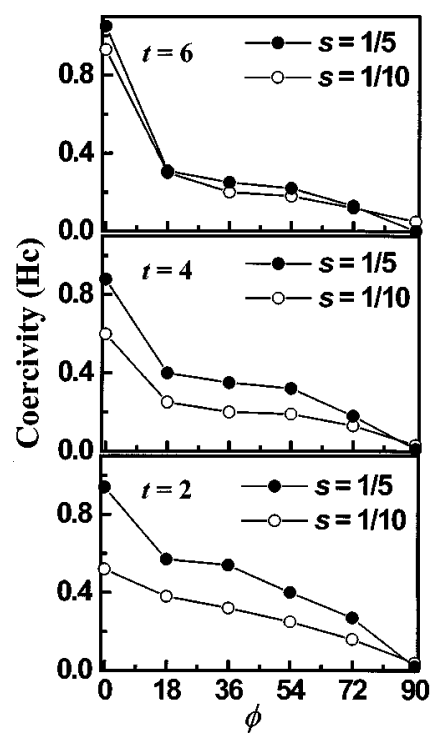

FIG. 3. Calculated coercive field as a function of $\phi$, showing its dependence on step densities for a fixed film thickness. 


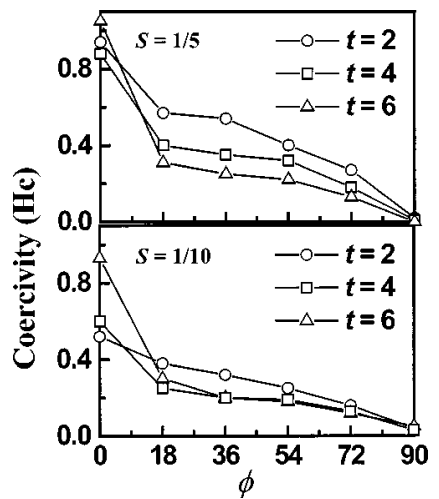

FIG. 4. Calculated coercive field as a function of $\phi$, showing its dependence on film thickness for a fixed step density.

The coercive field $H_{c}$ increases with increasing step density in all the films, consistent with experiments ${ }^{10}$ and previous theoretical calculations. ${ }^{16}$ Figure 3 also shows that the effect of step density is stronger in a thinner film than in a thicker film. For example, the coercive field at $\phi=0$ (with the external field along the step direction) increases substantially, from 0.52 to 0.94 , when the step density increases from $s$ $=1 / 10$ to $s=1 / 5$ in a thin, two-layer film (bottom panel in Fig. 3, $t=2$ ), but increases only slightly, from 0.93 to 1.05 , in a thicker, six-layer film (top panel, $t=6$ ). This behavior is consistent with the physical intuition that steps and surfaces play a less significant role in thicker films with more bulk spins. Quantitatively, in the thin samples $(t=2$ and $t=4)$, the coercivity increases on average by a factor of $\sim 1.6$ when the step density is doubled. Experimentally, Kawakami et al. ${ }^{10}$ show that the magnetic anisotropy in Fe films grown on stepped $\operatorname{Ag}(001)$ increases quadratically with increasing step density, while Jiang et al. ${ }^{7}$ show that the coercive field in $\mathrm{Co} / \mathrm{Cu}(001)$ films increases almost linearly with increasing step density. Simulations for a wider range of step densities must be done to determine the correct functional dependence of coercivity on step density.

Figure 4 shows the dependence of coercivity (as a function of $\phi$ ) on film thickness for a fixed step density, which exhibits a more complex behavior, as already evident from Fig. 3. Intuitively, we expect the coercive field to decrease with increasing film thickness, ${ }^{2}$ because the anisotropy energy is assigned only to the surface and step sites, and the ratio of the anisotropy energy to the total energy is proportional to the surface-to-volume ratio, which decreases with increasing thickness. This behavior is indeed obtained for a broad regime of large angles $\phi$ for which the external field lies closer to the direction perpendicular to the step direction. This is however, not the case when the direction of the external field is close to the step direction, i.e., along the easy axis. We speculate that such an anomalous behavior is caused by the detailed balance between the competing effects of the fourfold anisotropy assigned to the terrace sites and of the uniaxial anisotropy assigned to the step sites.

In addition to determining hysteresis loops, Monte Carlo simulation allows a direct recording and inspection of the surface spin configurations along every point in the hysteresis loops, and hence reveals directly the magnetization re-

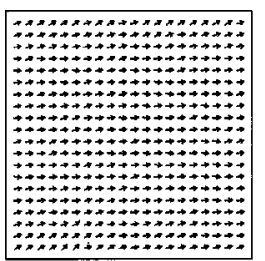

(a)

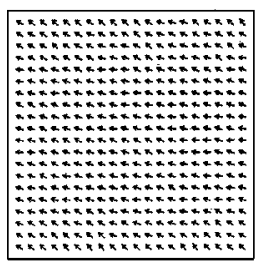

(d)

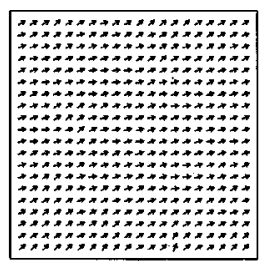

(b)

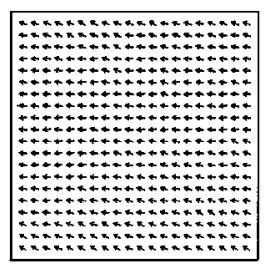

(e)

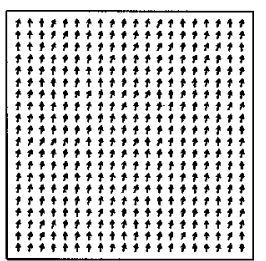

(c)

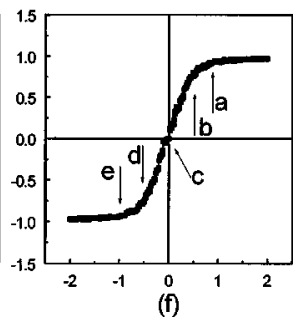

FIG. 5. Simulated equilibrium spin configurations for $t=4, s=1 / 5$, and $\phi=90^{\circ}$. (a) - (e) on (f) indicate the positions where the spin configurations are recorded in the hysteresis loop.

versal process. Figure 5 shows the spin configurations for the sample with $t=4, s=1 / 5$, and $\phi=90^{\circ}$ at five different positions in the hysteresis loop [see Fig. 5(f)]. The spin configurations show that the dominant mechanism for magnetization reversal is coherent rotation, in accordance with the model of Stoner and Wohlfarth. ${ }^{21}$ Hyman et al. ${ }^{16}$ have shown that different mechanisms for magnetization reversal may occur, depending on the parameters of anisotropy strength and step density. With the chosen anisotropy strength, all the systems that we simulated fall into the regime of the hysteresis phase diagram in which coherent rotation is the dominant mechanism for magnetization reversal, ${ }^{16}$ consistent with our direct inspection of spin configurations. The choice of our system parameters is likely to correspond to the real experimental values in certain systems. For example, Sussiau et $a l .{ }^{8}$ have concluded from the measured hysteresis loops of Co and FeNi films grown on step-bunched $\mathrm{Si}(111)$, in which a well-defined uniaxial anisotropy along the steps is expected, that a single-domain behavior with magnetization reversal by rotation is most likely.

In conclusion, we have investigated the hysteresis behavior for ultrathin magnetic films grown on a stepped surface, using Monte Carlo simulations. We have simulated the hysteresis loops as a function of the direction of the external field, focusing on the dependence of coercive fields on step densities and on film thickness. The coercive field decreases monotonically when the external field moves away from the step direction, the easy axis of the uniaxial anisotropy. In all the films studied, the coercive field increases monotonically with increasing step density, while its dependence on film thickness is more complex, decreasing monotonically with increasing thickness only when the external field direction is close to perpendicular to the steps. These observations can be generally understood in terms of the ratio between anisotropy energy, associated with the surface and step sites, and total energy, which is intrinsically linked to the surface/ volume ratio. They are also qualitatively in good agreement with experiments and previous theories. Direct inspection of spin configurations along the simulated hysteresis loops re- 
veals that the dominant mechanism for magnetization reversal is coherent spin rotation in all the systems we studied. Our studies indicate that in the design of ultrathin magnetic layers, the control of surface/interface morphology becomes increasingly more critical as the film thickness decreases, because the interplay between surface/interface roughness and film thickness becomes more prominent in thinner films.

This work was supported by AFOSR, Grant no. F4962095-1-0431.

${ }^{1}$ P. He, W. A. McGahan, S. Nafis, J. A. Woollan, Z. S. Shan, S. H. Liou, F. Sequeda, T. McDaniel, and H. Do, J. Appl. Phys. 70, 6044 (1991)

${ }^{2}$ H. Wan and G. C. Hadjipanayis, J. Appl. Phys. 70, 6059 (1991).

${ }^{3}$ J. Chen and J. L. Erskine, Phys. Rev. Lett. 68, 1212 (1992); J. Suen and L. Erskine, ibid. 78, 3567 (1997).

${ }^{4}$ H. P. Oepen, C. M. Schneider, D. S. Chuang, C. A. Ballentine, and R. C. O'Handley, J. Appl. Phys. 73, 6186 (1993).

${ }^{5}$ Y. L. He and G. C. Wang, J. Appl. Phys. 76, 6446 (1994).

${ }^{6}$ Q. Jiang, H. N. Yang, and G. C. Wang, Phys. Rev. B 52, 14911 (1995).

${ }^{7}$ Q. Jiang, H. N. Yang, and G. C. Wang, J. Vac. Sci. Technol. B 14, 3180 (1996).

${ }^{8}$ M. Sussiau, F. Nguyen-Va-Dau, P. Galtier and A. Schuhl, Appl. Phys. Lett. 69, 857 (1996)
${ }^{9}$ W. Weber, C. H. Back, A. Bischof, D. Pescia, and R. Allenspach, Phys. Rev. Lett. 76, 1940 (1996).

${ }^{10}$ R. K. Kawakami, E. J. Escorcia-Aparicio, and Z. Q. Qiu, Phys. Rev. Lett. 77, 2570 (1996).

${ }^{11}$ W. Wulfhekel, S. Knappmann, and H. P. Oepen, J. Appl. Phys. 79, 988 (1996).

${ }^{12}$ J. F. MacKay, C. Teichert, D. E. Savage, and M. G. Lagally, Phys. Rev. Lett. 77, 3653 (1996).

${ }^{13}$ A. S. Arrott, J. Appl. Phys. 69, 5212 (1991); A. S. Arrott and B. Heinrich, J. Magn. Magn. Mater. 93, 571 (1991).

${ }^{14}$ A. Moschel, R. A. Hyman, A. Zangwill, and M. D. Stiles, Phys. Rev. Lett. 77, 3653 (1996).

${ }^{15}$ M. Kolesik, M. A. Novotny, and P. A. Rikvold, Phys. Rev. B 56, 11791 (1997).

${ }^{16}$ R. A. Hyman, A. Zangwill, and M. D. Stiles, Phys. Rev. B 58, 9276 (1998), 60, 14830 (1999).

${ }^{17}$ D. Zhao, F. Liu, D. L. Huber, and M. G. Lagally, Phys. Rev. B 62, 11316 (2000).

${ }^{18}$ For a general review on magnetic anisotropy, see Ultrathin Magnetic Structures, Chapter 2, Vol. I, edited by B. Heinrich and J. A. C. Bland (Springer, Berlin, 1994).

${ }^{19}$ I. D. Mayergoyz, Mathmetical Models of Hysteresis (Springer, New York, 1991).

${ }^{20}$ C. Kawabata and K. Binder, Ann. Isr. Phys. Soc. 2, 988 (1978); M. Ferer, M. Moore, and M. Wortis, Phys. Rev. B 8, 5205 (1973).

${ }^{21}$ E. C. Stoner and E. P. Wohlfarth, Philos. Trans. R. Soc. London, Ser. A 240, 74 (1948). 